REVIEW

\title{
Intersex and gender assignment; the third way?
}

\author{
S F Ahmed, S Morrison, I A Hughes
}

Arch Dis Child 2004;89:847-850. doi: 10.1136/adc.2003.035899

The birth of a new baby is one of the greatest wonders of nature and one of the most exciting events known to man. The first question that is usually posed by the mother or father is "is it a boy or a girl?"; new parents cannot even formulate the second question which is usually "is he/she alright?". It is no wonder that the birth of a child with complex genital anomalies where the sex of rearing is uncertain at birth, presents difficult clinical and ethical issues.

See end of article for authors' affiliations

.....................

Correspondence to: Dr S F Ahmed, Consultant in Paediatric Endocrinology, Royal Hospital For Sick Children, Yorkhill, Glasgow G3 8SJ, UK;

gcl328@clinmed.gla.ac.uk

Accepted 31 January 2004
O ver the past few years, a number of justified concerns have been raised about the long term outcome of such infants and this has led to a comprehensive reappraisal of clinical management. While the current debate among clinicians, psychologists, ethicists, sociologists, historians, and patient support groups has long been overdue, the contribution of most of the participants has been largely based on personal experience and clinical evidence that often suffers from a substantial amount of selection bias and group based generalisation. There is a danger, however, that this debate may lead to a hiatus in providing optimal clinical care to the child and the child's family. Because sex, individual identity in most cultures, it is difficult to dislodge our ideas and, more so, our feelings about them, without a clear understanding of these issues. Delayed gender assignment of a newborn with complex genital anomalies is only one of the many issues under the spotlight and has often been muddled with another important question that relates to the timing of reconstructive surgery.

\section{GENDER}

In the first half of the twentieth century, the term gender was used to refer to those sexual behaviours that were more associated with men and women. Nowadays, gender relates to the social and cultural organisation of the sexes. Sex roles and gender roles used to be interchangeable terms but, increasingly, there is a difference between biological sex and social (gender) relations. It is one thing to have a vulva, vagina, clitoris, breasts, ovaries, 46XX karyotype, etc, but it may be quite another thing being female, feminine, or a woman. Unlike the sex categories, male and female, gender has several aspects: gender assignment, gender role, gender identity, gender attribution, and sexuality. In most societies, gender assignment occurs at birth, long before we have a say in the matter, marking the gender, and sexuality are at the very core of beginning of the process of gender socialisation. The process of gender socialisation also includes society's expectations of how males or females should behave, as expressed in their gender role behaviour. Gender identity is distinct from gender role behaviour and refers to the individual's perception of one's own gender and how it conforms to the male or female gender role in society. Gender attribution is what we all do when we meet someone and want to decide whether they are a man or a woman. This is often based on obtaining a number of cues which are symbolic manifestations of gender and that have traditionally included clothing, mannerisms, physical appearance, gait, and occupational choice. Finally, sexuality refers to erotic desires, sexual practices, or sexual orientation. In the Western culture, individuals are often socially identified as homosexuals or heterosexuals as if a person's sexual orientation encapsulates the total personality and identity. For most people, their gender identity, gender role, and the symbolic gender manifestations are congruent and, in addition, they will be sexually attracted to the opposite sex. However, it is also possible that a man may have gender manifestations that do not completely converge with his male gender identity and remains sexually attracted to the opposite sex; of course, a number of other permutations may also exist. Some aspects of gender, such as role, assignment, the symbolic manifestations, as well as the different types of sexuality, may differ markedly from one society to another and continue to evolve within respective societies. In some Western cultures, the distinction is becoming less absolute and it may be better to consider these aspects as a continuum, with female characteristics at one extreme and male ones at the other.

\section{GENDER DEVELOPMENT}

John Money proposed that we are born tabula rasa and our gender identity was programmed into us during the first few years of life, mainly through parental/social conditioning and reinforcement of gender role behaviour. ${ }^{1}$ It is possible that this original hypothesis may have contributed towards the rationale for performing early gender assignment and surgery in children presenting with complex genital anomalies at birth. This theory was famously challenged through the follow up of the John $v$ Joan case and by patient support groups, as well as subsequent research on long term outcomes..$^{2-4}$

The development of gender identity is the result of a complex interaction between genetic, prenatal, and postnatal endocrine influences and postnatal psychosocial and environmental experiences. However, Money's concept of a 
"critical period" for gender identity formation has remained a theme in current gender development research. Just as a child's cognitive and social development occurs in stages during the first five critical years of life, gender identity development is also often viewed according to age-stage/ cognitive development theory. ${ }^{5}$

Gender development research has focused on elements of gender identity formation such as gender knowledge, self perception, preferences (toy, playmate), and gender role behaviours. By the end of the first year of life, infants are already able to discriminate between the sexes, and some may be able to display sex related toy preferences. By 2-3 years of age children are able to correctly label themselves and others according to gender. By the age of 3 years, preference for one sex role has emerged with the child having a clear sense of whether he/she is a boy or girl. Children fix on cues such as clothing and hair in gender labelling exercises; even when genital cues are available they are used far less to make categorisation decisions than these other cues, at least until the age of 8 years or so, possibly reflecting insufficient biological understanding of gender differences.

By the age of 5 years, children learn that gender remains stable over time, becoming preoccupied with categorical differences between males and females. However, it is not until children have mastered the concept that gender remains constant (despite superficial changes in appearance), at the age of between 5 and 7, that many argue is when a gender identity has been fully attained. Theorists have suggested that once "gender constancy" has been mastered, this becomes a motivator to shaping sex appropriate gender behaviour. ${ }^{5}$

\section{PERCEPTION OF GENITALIA AND GENDER IDENTITY IN CHILDREN WITH COMPLEX GENITAL ANOMALIES}

There are scarce data on how children with complex genital anomalies perceive their genitals and how this relates to their gender development. Long term outcome case studies of such boys indicate that male gender identity does develop in the absence of gender specific genitalia. ${ }^{6}$ A systematic examination of the relation between genital appearance and gender identity in a group of girls with congenital adrenal hyperplasia, showed a link between atypical gender identity and prenatal androgen exposure, but not with the degree of virilisation. ${ }^{7}$ Taken together, this research suggests that genital appearance may not be as crucial a determinant in gender identity as once thought, although further research is necessary to clarify this observation.

\section{GENDER DIVERSITY}

In contemporary European (and North American) cultures, sex and gender have traditionally been based on binary opposites - male and female, man and woman, homosexual and heterosexual. There are a number of other cultures where there is greater gender diversity and gender does not always neatly divide into male and female. In these cultures, this gender variance has often been idealised and romanticised by many in the Western world. However, in such gender diverse cultures, the diversity always exists against a background of what it means to be male or female in that particular society. Social attitudes do vary within these cultures, as well as between them, and are complex. ${ }^{8}$ Nowadays, in most societies where these cultures exist, attitudes towards gender diversity include a combination of awe, fear, respect, ridicule, disgust, dismay, pity, and bemusement. Even when gender diversity is associated with ritual powers, such as on the Indian subcontinent, social interactions can often be hostile. Such attitudes and restricted occupational opportunities have led to segregation and development of subcultures and social communities. The degree to which this occurs probably depends on the size of the population; there is evidence to suggest that the acceptance of alternative genders may be easier in smaller, less socially specialised populations. ${ }^{8}$

\section{A POLICY FOR GENDER ASSIGNMENT}

The optimal policy for gender assignment needs to be a balance between our knowledge of the effect of the biological determinants that influence long term gender identity (that is, prenatal androgens) and the power of the affected individual in making decisions about his or her own outcome. Current evidence suggests that sex reassignment in adulthood is a relatively rare phenomenon and occurs rarely in children with undermasculinised boys ${ }^{9-11}$ and masculinised girls ${ }^{12} 13$ and when it does occur, it seems to occur most often in conditions associated with a defect in androgen biosynthesis which may result in spontaneous masculinisation at puberty. ${ }^{14}{ }^{15}$ Research in women with congenital adrenal hyperplasia suggests that although prenatal androgen excess may often lead to a masculinised gender role behaviour, this does not influence gender identity unless the affected individual presents late. $.^{12} 1316-19$ Recurrent genital reconstructive surgery may be associated with long term dissatisfaction with sexual function and an altered perception of body image, and it is possible that this may, itself, lead to a change in gender identity from female to male or vice versa. ${ }^{20}$ It is also possible that suboptimal medical therapy and a lack of knowledge about one's condition may have a greater effect on long term functional outcome than the original anatomic disorder. Genital masculinisation is a poor predictor of the masculinisation of the brain; not only is the correlation between these two poor, the underlying mechanisms are different whereby genital development is dependent on testosterone and dihydrotestosterone, while gender role behaviour is dependent on testosterone and oestradiol exposure. ${ }^{21}$ Although the John $v$ Joan case has been instrumental in challenging the management of children with complex genital anomalies, this single, rather atypical case of gender identity change from female to male gender may have over-exaggerated the role of prenatal hormones in the development of gender identity in all cases of complex genital anomalies. ${ }^{21}$ The gender assignment of the newborn with complex genital anomalies should not, therefore, just be based on a simple extrapolation of future gender role behaviour from the appearance of the genitalia at birth, but on the best prognosis for future psychosocial and psychosexual function, taking into account the clinical presentation of the child, details of the clinical syndrome, as well as the possible effects of prenatal steroid exposure. Given that gender role behaviour is a continuum, the possibility of altered gender role behaviour in the longer term should be recognised and discussed with the family, but it should not play a major part in influencing gender assignment at birth.

\section{COMPLEX GENITAL ANOMALIES}

Although genital anomalies such as hypospadias may have a birth prevalence of around 1 in 300 births, ${ }^{22}$ it is estimated that the birth prevalence of complex genital anomalies where gender assignment may be difficult at birth is about 1 in 4500 births. ${ }^{23}$ However, it is unclear how this figure has been derived, given that genital ambiguity depends on the clinical experience of the observer. Infants with complex genital anomalies due to 46XY undermasculinisation, but not those that result in completely female external genitalia (complete androgen insensitivity, testosterone biosynthetic defects) should usually be reared as boys. Although some of these infants will have dysgenetic gonads, a biosynthetic defect of testosterone production, or partial androgen insensitivity, in most of these cases the underlying biological abnormality 
remains unclear. These children will probably require a repair of the hypospadias, orchidopexy, and an evaluation of testicular function. In those cases where the testes are rudimentary and non-functional, it is advisable that they are removed as early as technically feasible. The next common clinical scenario is one of the masculinised, 46XX infant (for instance, congenital adrenal hyperplasia), and it is usually appropriate to raise these infants as girls. However, the decision in the severely masculinised infant may be influenced by factors such as a delay in diagnosis, social bias, and the "premium" on male rearing in certain communities. A modest degree of clitoral enlargement or genital ambiguity may not require surgical correction or aggressive suppression of serum androgen levels, and the emphasis should always be on long term functional outcome rather than cosmetic appearance. The need for an early genitoplasty in girls with marked clitoromegaly requires careful discussion and this should be documented in the medical records of the child. The surgeon should be allowed to retain the option of late reconstructive surgery for genitoplasty as well as vaginoplasty in those cases where it is likely that early surgery is more likely to be associated with long term adverse morbidity rather than improved functional outcome. Complex genital anomalies can also occur in children with gonadal dysgenesis associated with a $45 \mathrm{XO} /$ 46XY karyotype, and as most of these affected infants have a varying level of undermasculinised external male genitalia, it would be appropriate to raise most of these cases as boys. Although prenatal screening suggests that the incidence of $45 \mathrm{X} / 46 \mathrm{XY}$ may be about 1 per 15000 pregnancies, $90 \%$ of the offspring are phenotypically male. ${ }^{24}$ Gonadal differentiation in some of these cases may be mixed, and these infants would need to be approached as a true hermaphrodite where the infant may be $46 \mathrm{XX}, 46 \mathrm{XY}$, or $46 \mathrm{XX} / 46 \mathrm{XY}$ and possesses both ovarian and testicular tissue. ${ }^{25}$ This is a very rare condition estimated to have a population prevalence of about 15 per million inhabitants. ${ }^{26}$ As the reconstruction of the male genitalia had often been unsatisfactory in the past, gender assignment in these children had been biased towards the female sex. However, advances in reconstructive techniques for severe hypospadias and micropenis and concurrent reports of adequate long term satisfaction with sexual function in a substantial proportion of adults with micropenis is now leading to a reconsideration of this deep seated rationale. ${ }^{27}{ }^{28}$ Gender assignment in these cases will, therefore, be guided by the potential for future sexual and reproductive function and the potential for tumour development. Even in these complex cases, an adequate knowledge of the pathology and its long term outcome is sufficient to decide on gender assignment soon after birth.

\section{CONCLUSION}

Most clinicians agree that the goals of management in a newborn with complex genital anomalies should be the facilitation of a stable gender identity, provision of psychological support to the family, age appropriate education about the disorder, optimal sexual function, optimal body image, potential for reproduction, and minimal medical and surgical intervention. However, it is unrealistic to develop group based gross generalisations, especially as the priority assigned to these usual goals of management by the child, family, and carers will vary from case to case. A third gender is not a feasible option considering that in most cultures around the world, gender variants are not treated as equals and that the nations of the industrialised society are ill equipped to cope with this concept. Unless we decide that all individuals with complex genital anomalies live socially as "intersex people", this is not a simple solution and may be considered as "sweeping the problem under the carpet". Considering that, in most infants, the long term gender of rearing is not particularly disturbed, the attention needs to move away from the debate on delayed gender assignment to optimising surgical and medical intervention. Given that there is some evidence that early and multiple surgical interventions may be associated with adverse long term functional outcome, ${ }^{29} 30$ and there is little published evidence favouring these regimens, a careful multidisciplinary and fully informed discussion needs to be performed with the parents of an infant where surgery is contemplated. ${ }^{31}$ Surgery should only be performed by surgeons trained and experienced in genital reconstructive surgery and who are part of a multidisciplinary network concerned with a holistic management of the family and their child with complex genital anomalies. While polarised views exist about early versus late surgery in the clinical professions, each child and the respective family should be treated individually and the clinicians should refrain from imposing their own subjective views. The parents need to be provided with current objective evidence of effectiveness of the clinical interventions in optimising long term outcome, and they should be made aware of current knowledge about gender development. Irrespective of the parents' decision, health professionals should be prepared to provide the appropriate psychological, surgical, and medical support to them as well as their affected child. Sufficient resources need to be available to support clinical networks that encourage these activities, and which can audit these processes and develop suitable guidelines, so that we will not still be in the same position in another three to four decades.

\section{ACKNOWLEDGEMENTS}

SM is supported through the Scottish Audit of Genital Anomalies, a three year national project funded by NHS Quality Improvement Scotland.

\section{Authors' affiliations}

S F Ahmed, S Morrison, Scottish Genital Anomaly Network, Scotland, UK

I A Hughes, Dept of Paediatrics, University of Cambridge, UK

This paper is dedicated to the memory of Sita Picton, Clinical Psychologist at the Royal Hospital For Sick Children, Yorkhill, Glasgow.

\section{REFERENCES}

1 Money J, Hampson JG, Hampson JL. Imprinting and the establishment of gender role. Arch Neurol Psychiatry 1957;77:333-6.

2 Diamond M, Sigmundson HK. Sex reassignment at birth: a long term review and clinical implications Arch Paediatr Adolesc Med 1997;151:298-304.

3 Chase $\mathbf{C}$. What is the agenda of the intersex patient advocacy movement? Endocrinologist 2003;13:240-2.

4 Diamond M, Sigmundson HK. Sexual identity and sexual orientation in children with traumatised or ambiguous genitalia. J Sex Res 1997;34:199-211.

5 Ruble DN, Martin CL. Gender development. In: Damon W, Eisenberg N, eds. The handbook of child psychology, 5th edn. Vol. 3. Social, emotional and personality development. New York: Wiley, 1998.

6 Zucker K. Intersexuality and gender identity differentiation. Ann Rev Sex Res 1999; 10:1-69.

7 Berenebaum S, Bailey MJ. Effects on gender identity of prenatal androgens and genital appearance: evidence from girls with congenital adrenal hyperplasia. J Clin Endocrinol Metab 2003;88:1 102-6.

8 Nanda S. Gender diversity: cross-cultural variations. Illinois: Waveland Press, 2000.

9 Sandberg DE, Meyer-Bahlburg HF, Yager TJ, et al. Gender development in boys born with hypospadias. Psychoneuroendocrinology 1995;20:693-709.

10 Hines M, Ahmed SF, Hughes IA. Psychological outcomes and gender-related development in complete androgen insensitivity syndrome. Arch Sex Behav 2003:32:93-101.

11 Wisniewski AB, Migeon CJ. Long-term perspectives for 46, XY patients affected by complete androgen insensitivity syndrome or congenital micropenis. Semin Reprod Med 2002;20:297-304

12 Berenbaum SA, Bailey JM. Effects on gender identity of prenatal androgens and genital appearance: evidence from girls with congenital adrenal hyperplasia. J Clin Endocrinol Metab 2003;88:1102-6.

13 Slipper FM, Drop SL, Molenaar JC, et al. Long-term psychological evaluation of intersex children. Arch Sex Behav 1998;27:125-44. 
14 Mendez JP, Ulloa-Aguirre A, Imperato-McGinley J, et al. Male pseudohermaphroditism due to primary 5 alpha-reductase deficiency: variation in gender identity reversal in seven Mexican patients from five different pedigrees. J Endocrinol Invest 1995;18:205-13.

15 Mendonca BB, Inacio M, Arnhold IJ, et al. Male pseudohermaphroditism due to 17 beta-hydroxysteroid dehydrogenase 3 deficiency. Diagnosis, psychological evaluation, and management. Medicine 2000;79:299-309.

16 Berenbaum SA. Effects of early androgens on sex-typed activities and interests in adolescents with congenital adrenal hyperplasia. Horm Behav 1999;35: 102-10

17 Hines M, Kaufman FR. Androgen and the development of human sex-typical behavior: rough-and-tumble play and sex of preferred playmates in children with congenital adrenal hyperplasia (CAH). Child Dev 1994:65: 1042-53.

18 Woelfle J, Hoepffner W, Sippell WG, et al. Complete virilization in congenita adrenal hyperplasia: clinical course, medical management and diseaserelated complications. Clin Endocrinol 2002;56:231-8.

19 Kandemir N, Yordam N. Congenital adrenal hyperplasia in Turkey: a review of 273 patients. Acta Paediatr 1997:86:22-5.

20 Reiner WG, Gearhart JP. Discordant sexual identity in some genetic males with cloacal exstrophy assigned to female sex at birth. N Engl J Med 2004;350:333-41.

21 Hines M. Abnormal sexual development and psychosexual issues. Baillieres Clin Endocrinol Metab 1998;12:173-89.
22 Ahmed SF, Dobbie R, Finlayson AR, et al. Regional and temporal variation in the occurrence of genital anomalies amongst singleton births, 1988-1997, Scotland. Arch Dis Child Fetal Neonatal Ed 2004;89:F149-51.

23 Sax L. How common is intersex? A response to Anne Fausto-Sterling. J Sex Res 2002:39:174-8.

24 Chang HJ, Clark RD, Bachman $H$. The phenomenon of $45 X / 46 X Y$ mosaicism: an analysis of 92 prenatally diagnosed cases. Am J Hum Genet 1990:46:156-67.

25 Telvi L, Lebbar A, Del Pino O et al. 45,X/46, XY mosaicism: report of 27 cases. Pediatrics 1999;104:304-8.

26 Kuhnle U, Krob G, Maeir E. True hermaphroditism: presentation, management and outcome. Endocrinologist 2003;13:214-18.

27 Reilly JM, Woodhouse CR. Small penis and the male sexual role. J Urol 1989;142:569-71.

28 Wisniewski AB, Migeon CJ, Gearhart JP, et al. Congenital micropenis: long term medical, surgical and psychosexual follow-up of individuals raised male or female. Horm Res 2001;56:3-11.

29 Creighton SM, Minto C, Steele S. Objective cosmetic and anatomica outcomes at adolescence of feminising surgery for ambiguous genitalia done in childhood. Lancet 2001;358:124-5.

30 Minto CL, Liao LM, Woodhouse CR, et al. The effect of clitoral surgery on sexual outcome in individuals who have intersex conditions with ambiguous genitalia: a cross-sectional study. Lancet 2003;361:1252-7.

31 Cull M. Treatment of intersex needs open discussion. BMJ 2002;324:919

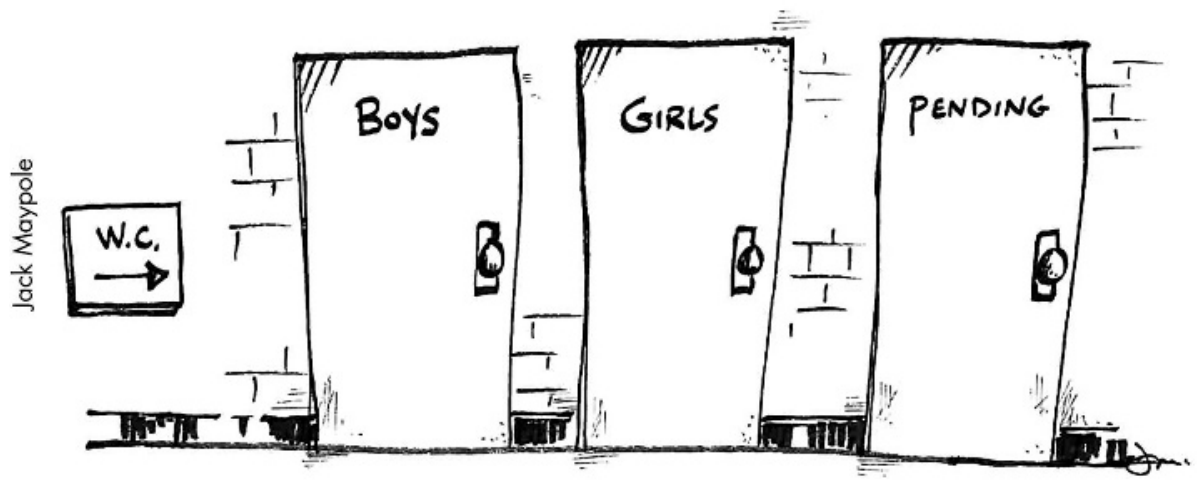

